Measurement

Manuscript Number: MEAS-D-18-01791R1

Title: Laser diodes optical output power model

Article Type: Research Paper

Keywords: Laser diodes optical model, Pspice model, Modelling method, Characterization system

Corresponding Author: Mr. RAMON BORRAS, Mr.

Corresponding Author's Institution: Monocrom S.L.

First Author: RAMON BORRAS, Mr.

Order of Authors: RAMON BORRAS, Mr.; Joaquin del Rio; jordi juliachs; carles oriach; joan montiel

Abstract: This article proposes a modelling method of laser diodes optical output power, especially for mid-high power diodes working in continuous wave (CW) or quasi continuous wave (QCW) modes, and focusing in the optical output power dependency on temperature and its model implementation in Pspice, targeting its computer simulation. It is commented the theory and related mathematical expressions used in the model, the Pspice program model and the purpose of its different parts, and how mathematical expressions are introduced in it. It is proposed a diodes optical output power response characterization method using an automatic data acquisition system to obtain diodes response curve avoiding temperature effects during the measurements. Implementation example of the modelling and characterization method in a laser diode, model results versus real measurements comparison and related conclusions are exposed. 


\title{
Laser diodes optical output power model
}

\author{
Ramon Borràs ${ }^{1,2}$, Joaquin del Rio ${ }^{1}$, Carles Oriach ${ }^{2}$, Jordi Juliachs ${ }^{2}$, Joan Montiel $^{2}$ \\ ${ }^{1}$ Electronics Department, Universitat Politècnica de Catalunya,Vilanova i la Geltrú, Spain \\ ${ }^{2}$ Monocrom S.L., R\&D Department, Carrer Vilanoveta, 6, 08800, Vilanova i la Geltrú, Spain \\ E-mail: r.borras@monocrom.com
}

\begin{abstract}
:
This article proposes a modelling method of laser diodes optical output power, especially for mid-high power diodes working in continuous wave $(\mathrm{CW})$ or quasi continuous wave $(\mathrm{QCW})$ modes, and focusing in the optical output power dependency on temperature and its model implementation in Pspice, targeting its computer simulation. It is commented the theory and related mathematical expressions used in the model, the Pspice program model and the purpose of its different parts, and how mathematical expressions are introduced in it. It is proposed a diodes optical output power response characterization method using an automatic data acquisition system to obtain diodes response curve avoiding temperature effects during the measurements. Implementation example of the modelling and characterization method in a laser diode, model results versus real measurements comparison and related conclusions are exposed.
\end{abstract}

Keywords: Laser diodes optical model, Pspice model, Modelling method, Characterization system

\section{1- Introduction}

Modelling need of laser diodes optical output power is related to the fact that the device temperature is increasing due to the laser effect and decreasing the optical output power. This effect is especially important when the laser diode device is used in continuous or quasi continuous wave modes. Most of the laser diodes applications are based in a constant optical output power and in order to achieve it two options are used: either to keep constant the temperature or to use feedback circuits readjusting the injected current [1]. In the first case, even though the device temperature is kept constant by using temperature conditioning systems like Peltier cells or chiller circuits, it is necessary to consider that it may exist a temperature gap between the semiconductor's $\mathrm{p}$-n junction and temperature control elements inside the device or the system like thermistors or thermometers. This gap should be measured, for instance based on the emitted light spectrum using spectrometers, to evaluate the necessary margins to keep the device inside operational limits. In the second case, feedback circuits based on photodiodes measurement, compensating the optical output power decrease with more injected current, will tend to increase the temperature of the laser diode device up to an unknown value that will depend on the overall system temperature dissipation capability. In both cases, constant temperature or feedback circuits, it is worth to simulate device response at different temperatures assuring its usage inside operational limits to avoid possible device damages. Laser diodes are the most robust of the laser devices, but temperature and current limits should be respected.

The laser diodes optical power output dependency on temperature is a known effect. Information of how this temperature variation works and related theory are available [2] and it is used for this methodology proposal. The novelty of this work is to adopt some hypothesis to complete the temperature dependency mathematical expressions and to define a modelling method targeting this temperature dependency to be programmed in a computer simulation tool like Pspice environment. This includes:

- $\quad$ to identify which are the required parameters and how to obtain their values to propose the mathematical expressions for the temperature dependency

to propose a Pspice simulation model schema

to include the obtained mathematical expressions in the simulation schema and obtain its Pspice programmed model, and to define a temperature independent measurement method including testing signal conditions and measurement tools to properly achieve the correct parameters values required for the model.

This last item refers to the so-called cold measurement method and the characterization system setup. This setup includes developing an automatic data acquisition system to measure the relation between the optical output power Popt, the laser diode current Ild and the temperature $\mathrm{T}$, to be used with temperature conditioning systems like chillers circuits or climatic chambers.

To be able to define the temperature dependency model of the optical output power of a laser diode with this proposed modelling method it is necessary to know how is the variation of two parameters of the optical response with the temperature, the threshold current $\mathrm{I}_{\mathrm{th}}$, minimum injected current to start optical emission, and the slope efficiency SE or differential efficiency $\eta_{\mathrm{D}}$, defined as the relation between the optical output power and the injected current increments. Some assumptions or hypothesis are taken related to the variation of the slope efficiency with the temperature. No information about this variation has been found in the state of the art of the theory, so this is also a novelty of this work. This hypothesis is based on some laser diode devices specification data, and it is confirmed with results obtained in a climatic chamber. Those results also confirm the variation of the threshold current with the temperature as exposed in the theory. 
The selected electronic circuits simulation tool in this work is Pspice and the programmed models are developed and simulated using it. Even thought the mathematical model and expressions are of course general and could be used to program any other simulation tool. The modelling method proposed in this article has been applied to different output power ranges laser diodes devices of different wavelengths, from $5 \mathrm{~mW}$ diodes up $40 \mathrm{~W}$ laser bar diodes. Model has been validated by comparison of simulation results and measured values in all of them. In this article, as a use case example, the overall modelling method and characterization system are mainly applied to a concrete diode, an $8 \mathrm{~W}$ single emitter laser diode, even some references to other laser diodes are sometimes done to help the explanation of certain concepts.

The structure of the article is as follows:

- Section 1, the Introduction where motivation and overall view of the article's contents is explained.

Section 2 is a state of the art explanation regarding laser diodes modelling methods

In section $\underline{3} z$ it is explained the laser diode model, both electrical and optical output power models. This includes their parameters, how to obtain their values and how the models are depending on the temperature. Also related adopted hypothesis.

| - In section $\underline{43}$, Pspice model is proposed, which includes the Pspice model schema and their components, how those are used and their purpose, the used existing Pspice model libraries and components and how the temperature dependency is introduced in the model, together with the mathematical model expressions of the optical output power.

- Section 54 is about the characterization proposed method, with the so-called cold measurement method, the measurement setup environment, the automatic acquisition system, how are the optical output power parameters obtained, the hypothesis validation with obtained results and finally the programmed Pspice model of the laser diode and its validation by simulated and measured results comparison.

And finally, in Section $\underline{6} 5$ conclusions of the overall research exposed in the article are commented.

Regarding the measurement field, and compared with other explained methodology for laser diodes optical output power modelling, the proposed methodology is based on the measurement of the laser diodes optical response. The most important challenge faced during this research is how to measure the temperature related parameters on a laser diode device due to the fact that its temperature increases when the current is injected in it just for the intended measurement itself. It is known that at least the $50 \%$ of the energy injected in the laser diode device is converted in heat and increases device's temperature. Even using temperature conditioning systems, it may exist a gap between the semiconductor p-n junction and the temperature measurement element in the device, normally a thermistor, or in the measurement system, normally a thermometer close to the device measuring the temperature of the refrigeration fluid. Problem faced is that parameters that define the temperature behaviour and its model have to be measured at a constant temperature, during a current level range which needs to be wide enough to be able to obtain the full optical output power response characteristic. But the current through the device could modify the temperature during the measurement. In order to overcome this problem it has been defined a cold measurement method that consists in:

- acquiring necessary measurement data during a very short rising up time current slope, short enough to avoid any temperature increase during that data acquisition

- dismiss any data acquired during the rest of the injected current pulse, as it could be theoretically more affected of a temperature increase

- control the p-n junction temperature during the measurement, by means of supervising the wavelength spectrum, and assuring that there is no temperature drift during the measurement

Short rising up time current slopes of $20 \mu$ s have been used with the proper drivers. This means that fast data acquisition cards with synchronised triggers and independent data channels have been used. Combined with spectrometers usage, a reliable temperature independent measurement method of the laser diode optical output power response characteristic is achieved, as it will be commented in the characterization method section.

Existing methodology for laser diodes optical output power response characterization based in its response consists in measuring the optical output power response level to injected current pulse of different levels, obtaining different points of the optical output power response slope to define it. Those pulses are intended to be as short as possible, also to avoid as much as possible any temperature increase in the measurement. These series of pulses need to be repeated at every temperature the characteristic need to be measured at. The proposed method is measuring the whole optical output power characteristic as a response to a single short rising up current slope. The clear advantage of this new methodology is that only one current slope is necessary to obtain the full response characteristic at each temperature. The measurement equipment needs also to be more complex, using fast data acquisition cards with independent channels and trigger inputs instead of oscilloscopes. 


\section{2- State of the Art}

The main objective of this work is to define a modelling method of laser diodes behaviour including their temperature dependency and to program the related model in Pspice, targeting commercial mid-high power laser diodes working in continuous or quasi continuous wave modes. Information about laser diodes modelling methods can be found in several articles and doctoral thesis.

\subsection{Rate equations based modelling method}

Several articles and doctoral thesis are proposing modelling methods of the laser diodes based in the so called Rate Equations and the resultant equivalent electrical circuits that could be used for Pspice model programming. Those rate equations describe the variation of carriers and photons densities in the semiconductor. Equivalent electrical circuits are proposed from the resultant voltages and currents, that can be used to program a model in a simulation tool. Rate equations are depending on the physical characteristics of the diodes like the laser cavity volume, diode's topology, for instance if it is a quantum well (QW) or multi quantum well (MQW) diode, and other parameters like the semiconductor doping or the average recombination lifetime of photons and carriers. That is why in some articles the model obtained from the Rate Equations is called Physic-Based model. Different equivalent electrical circuit models are obtained based on those parameters. It is important to note that to be able to obtain the equivalent circuit model based on the Rate Equations it is necessary to have a detailed information of diodes physical parameters, which is not always possible in case of commercial laser diodes which are the target of this research. Available information from manufacturers of commercial diodes is normally limited. Rate Equations modelling method is very useful in case of new laser diodes topology studies, but not very convenient in case of commercial devices. Some examples of Rate Equation modelling based of multi quantum well laser diodes are the articles A PSPICE Circuit Modelling of Strained AlGaInN Laser Diode Based on the Multilevel Rate Equations [24], Modelling Quantum Well Lasers [25], and the doctoral thesis Wide-Band and Scalable Equivalent Circuit Model for Multiple Quantum Well Laser Diodes [26]. In all of them an equivalent electrical circuit is obtained from the Rate Equations, and in all of them a full list of required parameters values are necessary to obtain the equivalent electrical circuit component values. Some examples of the same method applied in case of Double Heterojunction Laser Diodes (DHLD) are the doctoral thesis Circuit-Level Modeling of Laser Diodes [28] and the article Double Heterojunction Laser Diode (Tucker Model) DHLD [29], both from same author, where Rate Equations and V/I characteristics of the laser diodes are used to obtain the equivalent electrical circuits for simulation programming. Also in the article The complete electrical equivalent circuit of a double heterojunction laser diode using scatterring parameters [30] the so called single-mode rate equations are used to obtain the equivalent electrical circuits for both, the intrinsic circuit of the diodes and the complete circuit including parasitic effects from the case and electrical contacts. In all of them again a full list of parameters values are necessary. Other examples of the rate equations based method applied to other diodes topologies are the articles Modelización y Simulación de dispositivos optoelectrónicos utilizando el programa SPICE [27] applied to a Fabry-Perot laser diode and the article Theoretical Model of bistable Semiconductor Laser Diode Based on the Rate Equations [31] and the doctoral thesis Circuit-Level Modeling and Simulation of Semiconductor Lasers [32].

\subsection{Response curves based modelling method}

A more convenient method for industrial purpose of modelling laser diodes behaviour and its dependency on temperature is from their response curves, it means based on the measured relation between the optical output power and the injected current in the laser diode at different temperatures, and using those response curve results to define the laser diodes behaviour as a part of the Pspice model to be programmed. This method has clear advantages in the industrial use. A disadvantage is that proper electronic drivers are necessary to control the injected current, adapted to each laser diode. Advantages are, the laser diode is measured as a black box including in its measurement all effects due to laser emission and optical efficiencies. It is not required a detailed knowledge of the physical parameters of the laser diode and its semiconductor as in the previous rate equation based case, which allows an easier implementation of a practical modelling method for commercial devices to be used in the industrial field. An example of using this modelling method based on the laser diode response curves is the article Simple Spice model simulates laser diode [33]. In this article, laser diode's optical output power versus injected current relation and the associated photodiode's current versus optical output power response, are used to define a equivalent electrical circuit to be simulated in Spice, finally including also the Spice program or netlist of the laser/monitor diode pair.Another example of this methodology is the article Modelling Lasers with IsSpice and PreSpice [34]. In this article, Hitachi's HL7801E diode is modelled as a GaAlAs laser diodes example. Model is done from the response curves provided by manufacturer in its specification. Also finally the model is programmed in Spice.

There are already existing some examples of Pspice implementation of laser diodes, some of them directly provided in Pspice libraries, even none of them are including the temperature variation effect in the model. Examples are the SLD1121VS, the SLD1132VS and the SLD323V from SONY. In all of them the model includes a photodetector diode. In the current state of the art there are not a Pspice or any other simulation tool model which includes the optical output power variation with the temperature. This will be the main challenge of this study, the proposal of the mathematical expressions, the required parameters to define the temperature dependency expressions, the temperature dependent expressions introduction in the Pspice model, and the proposal of measurement method for those parameters to avoid any temperature variation effect during their measurement.

Formatted: Justified, Space After: 0 pt 


\section{2- Laser diodes electrical and optical modelling}

\section{1- Laser diodes electrical modelling}

Modelling the laser diode optical output power will only consider forward bias where the laser effect takes place. In this area, the electrical model of a diode is well known and follows the Shockley equation [3][4]:

$$
I d \approx I s \cdot e^{\left(\frac{V d-I d \cdot R s}{N \cdot V t}\right)}
$$

$$
V d=\ln \left(\frac{I d}{I s}\right) N \cdot V t+I d \cdot R s
$$

where Id and Vd are the current and voltage at the diode, Vt is the thermal voltage, Rs the parasitic resistance, Is the saturation current and $\mathrm{N}$ the ideality factor, also known as quality factor or emission coefficient.

Electrical behaviour is temperature dependent as:

$$
I \boldsymbol{S}(\boldsymbol{T})=I \boldsymbol{S} \cdot \boldsymbol{e}^{\left[\left(\frac{T}{T \text { nom }}-1\right)\left(\frac{E G}{N \cdot V t}\right)\right]} \cdot\left(\frac{T}{T \text { Tnom }}\right)^{\left(\frac{X T L}{N}\right)}
$$

where Is is the saturation current at the nominal temperature Tnom, EG the Energy Gap and XTI the saturation current temperature exponent.

\subsubsection{Laser diode electrical model parameters}

Rs and Is can be calculated with values obtained from the measured I/V response curve at a certain temperature applying (1). Fig.1 is an example of the measured I/V chart at forward bias at $25^{\circ} \mathrm{C}$ of a $808 \mathrm{~nm}$ wavelength emitted light laser diode of $8 \mathrm{~W}$ of optical output power. It is the WSLX808008 single emitter laser diode from Wavespectrum [6]. Values of the I/V response have been obtained in CW, using a control driver, the DRVx2PITEC of MONOCROM S.L [7][8], controlling with it the current through the laser diode and measuring the voltage at diode's terminals in forward bias. This chart therefore corresponds to the Shockley equation (1). Values of the attached table have been used to calculate Is and Rs parameters.

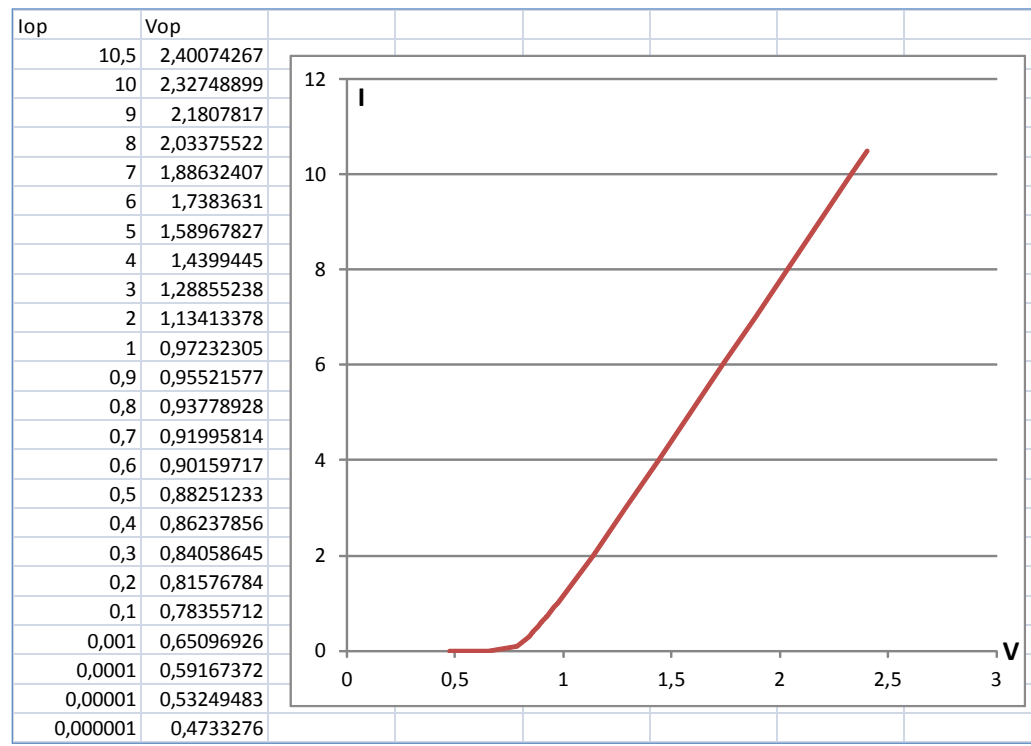

Fig.1: Measured I/V response curve of the WSLX808008 laser diode at 25 으

In a good diode and in forward bias operation it can be considered N=1 [3]. It is to be used in Is and Rs calculation using (1). Related to the electrical temperature dependency parameters (2), EG value depends on semiconductor material's alloy composition and therefore related to the emitted light wavelength. It can be obtained from tables or charts like Fig. 2 [9] where it is shown an example of EG value, obtained based on the laser diode wavelength emitted or the semiconductor alloy composition. In the WSLX808008 laser diode, it is a $808 \mathrm{~nm} \mathrm{AlGaAs}$ laser diode, and therefore its alloy composition is $\mathrm{Al} 10 \%$, Ga $90 \%$. Its EG value in the chart is $\mathrm{EG}=1.55$ $(\mathrm{eV})$. Related to parameter XTI, XTI $=3$ is a known value in a p-n semiconductor diode and this value is used as default in the Pspice model [5]. Expressions (1) and (2) are already implemented in the used Pspice diode model, so introducing those values for those parameters in the model will directly allow its simulation. 
In the example of $\mathrm{I} / \mathrm{V}$ curve shown in Fig.1, applying (1) with $\mathrm{N}=1$, Is at $25^{\circ} \mathrm{C}$ is calculated, and applying (2) with EG and XTI commented values, the Is value at $27^{\circ} \mathrm{C}$, which is the value to be used in the Pspice model as it is explained in the following section 3 . Obtained values to be introduced in the model are Is $\left(27^{\circ} \mathrm{C}\right)=1.525 \mathrm{E}-14 \mathrm{~A}$ and $\mathrm{Rs}=0.144 \Omega$.

Measuring I/V response curves of the diode at different temperatures and applying (1) and (2), values of Is (T) and Rs can be obtained, and from those, EG and XTI parameters can be also calculated using (2). This would be an alternative method for EG and XTI calculation if those parameters are not known beforehand and without need of knowing the emitted wavelength or the semiconductor alloy composition.

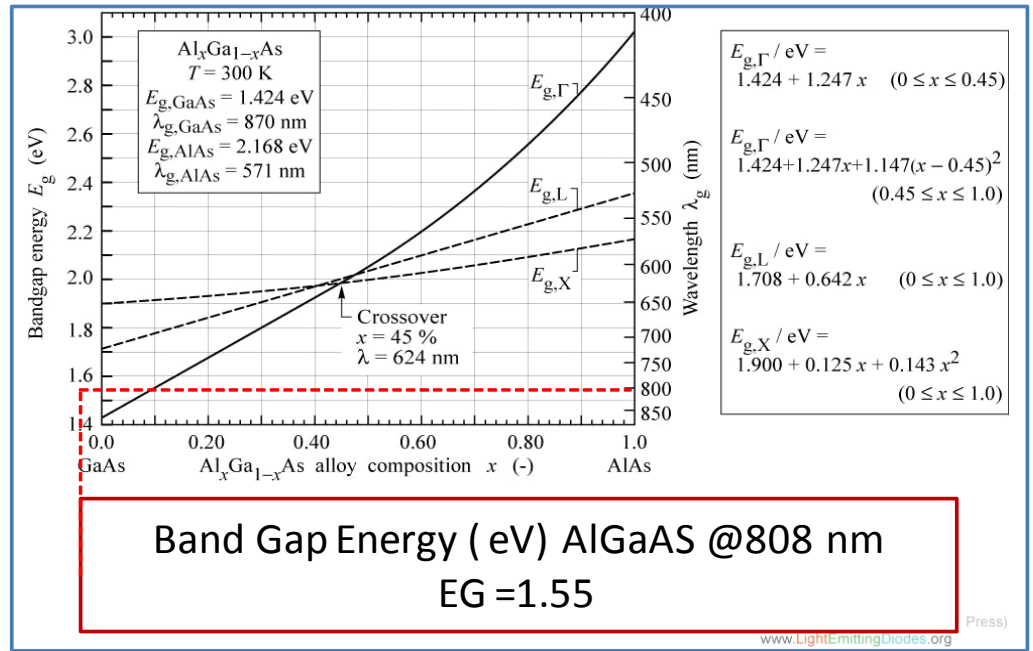

Fig.2: EG value / wavelength or alloy composition [9]

\section{2- Laser diodes optical output power modelling}

\subsection{1- Laser diode optical output model}

Referring to theory exposed in [2], the proposed optical model for the Popt/Ild of a laser diode (optical output power vs. laser diode current) is:

$$
\text { Popt }=\text { 0, for Ild } \leq \text { Ith; }
$$$$
\text { Popt }=S E \cdot(I l d-I t h) \text {, for Ild } \geq I t h
$$

where $I_{t h}$ is the threshold current laser effect and emitted light start from, and where SE is the slope efficiency, also known as Differential efficiency $\eta_{D}$, which is the relation between the optical output power and diode's current increments. This expression (3) defines a simple model based in two lines able to be easily programmed including the temperature variations.

\subsubsection{2- Model variation with temperature}

Fig. 3 shows an example of how this model is implemented and its variation with the temperature. In this case, the model is based on data obtained from a low power laser diode of $670 \mathrm{~nm}$ of emitted wavelength, and typical operation values of $5 \mathrm{~mW}$ of optical output power at $50 \mathrm{~mA}$ of laser diode current working at $25^{\circ} \mathrm{C}$ [10]. The shown chart is the simulation result in Pspice program. Low power laser diodes have wider temperature operational ranges, in this case from $-10{ }^{\circ} \mathrm{C}$ up to $60^{\circ} \mathrm{C}$. This low power diode example is therefore used to better illustrate the temperature dependency of related parameters $\mathrm{I}_{\text {th }}$ and SE.

In the proposed Popt $(\mathrm{T})$ model the value of the threshold current Ith is increasing with the temperature increase and the value of the slope efficiency SE is decreasing. Ith variation with temperature has a known behavior as expression :

$$
\operatorname{Ith} 2=I \operatorname{th} 1 \cdot e^{\frac{T 2-T 1}{T 0}} \quad \text { or } \quad \operatorname{Ith}(T)=I t h 1 \cdot e^{\frac{T-T 1}{T 0}}
$$

where $\mathrm{I}_{\text {th } 1}$ is $\mathrm{I}_{\mathrm{th}}$ at temperature $\mathrm{T}_{1}$ and $\mathrm{I}_{\mathrm{th} 2}$ is $\mathrm{I}_{\mathrm{th}}$ at temperature $\mathrm{T}_{2}$. To parameter value depends on semiconductor type. As an example, value for InGaAsP laser diodes is between 40 and $75 \mathrm{~K}$ [2].

As for the slope efficiency SE, based on some laser devices specifications like [11] or [12] of $5 \mathrm{~mW}$ and $1 \mathrm{~W}$ optical output power, the hypothesis is that SE value will decrease linearly with the temperature within the operational working limits. This hypothesis will be confirmed with real measurements in a climatic chamber with the $8 \mathrm{~W}$ laser diode as explained in following sections. Based on it, the expression of the SE variation with the temperature is:

$$
S E(T)=S E 1+(T-T 1)\left(\frac{S E 2-S E 1}{T 2-T 1}\right)
$$


where $\mathrm{SE}_{1}$ and $\mathrm{SE}_{2}$ are $\mathrm{SE}$ values at temperatures $\mathrm{T}_{1}$ and $\mathrm{T}_{2}$. Replacing (4) and (5) in (3) the model expression of the optical output power variation with the temperature is:

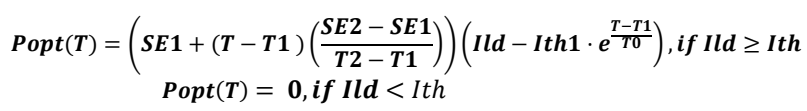

Value of To is obtained from (4) as:

$$
T o=\frac{T 1-T 2}{\ln I t h 1-\ln I t h 2}
$$

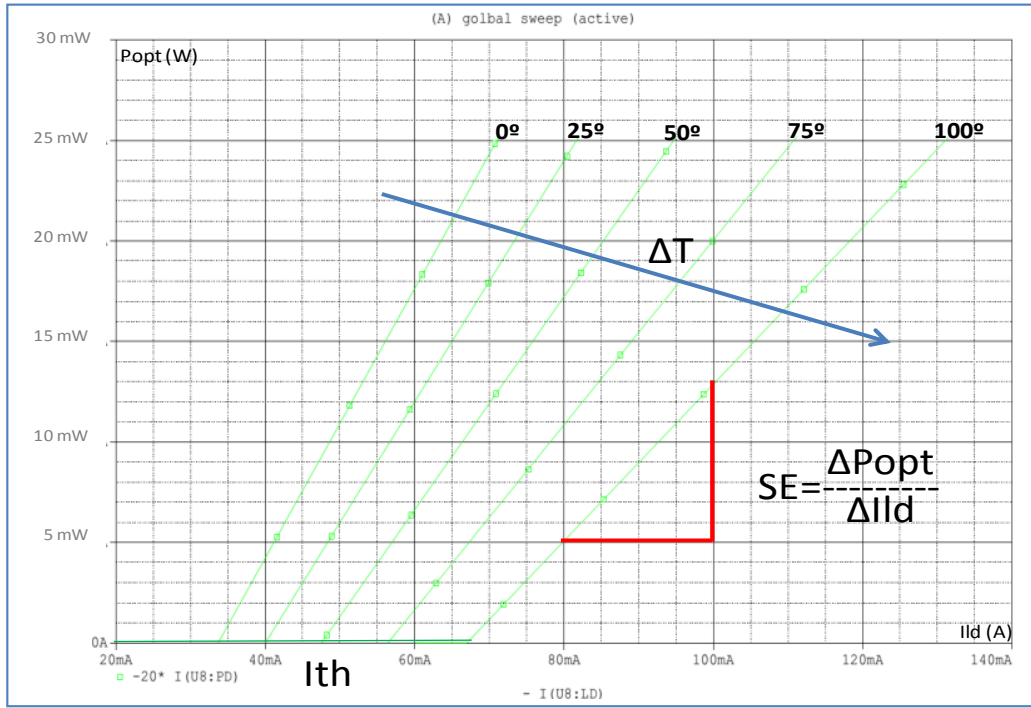

Fig.3: Popt(T) model

\section{3- Popt(T) Pspice model of a laser diode}

\section{1- Laser diode Pspice model schema}

The proposed Pspice model of a laser diode is based on the schema shown if Fig.4:

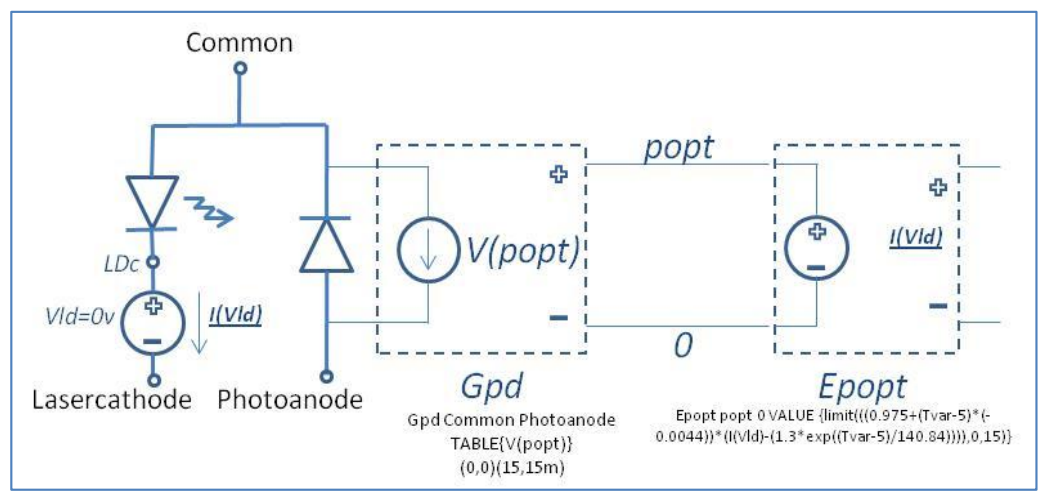

Fig.4: Popt(T) Pspice model schema of a laser diode

In this schema the laser diode's cathode LDc is connected to a source which current I(Vld), the current through the laser diode, will be used to model the optical output power. It is done using a voltage controlled voltage source (Epopt). In this source, the control voltage value is the laser diode current I(Vld) and the output voltage V(popt) is the value of the optical output power Popt(T) (6). It is therefore in Epopt source expression where the optical output power $\operatorname{Popt}(\mathrm{T})$ due to the current through the laser diode I(Vld) can be introduced including its temperature dependency. Gpd is a voltage controlled current source used to linearly convert the optical output power 
value represented by $\mathrm{V}$ (popt) into a current through a photodiode, as a way to electrically measure the optical output power in the model. The photodetector diode is in reverse bias and could be deleted, as all current through terminals Common and Photoanode in forward bias, where optical power is measured, is due to Gpd current source. Photodetector diode is kept in the schema to help its functionality understanding. The relation between the optical output power and the current through the diode is the sensitivity of the photodiode. It is necessary to know which is this relation in case of existing a real photodiode to assign the proper optical power to the current measured in it. The output current of Gpd is therefore connected to the terminals of the photodiode to force in it this current value. In this schema the laser diode and the photodiode are sharing a Common terminal, laser anode and photodiode cathode, in a called common anode configuration. The schema could have been proposed with separated laser diode and photodiode or even with different laser and photodiodes common terminal configurations.

\section{2- Pspice program. Used available models and values}

For the laser diode it is used the diode's Pspice model available in the libraries, which is already including the electrical response and therefore the forward bias Shockley equation (1) and the temperature dependency of the saturation current Is (2). It will be necessary to introduce the proper parameters as explained in section 1. In Pspice the nominal temperature Tnom is $27^{\circ} \mathrm{C}$, so it is necessary to obtain Is at that temperature or calculate it using (2) if Is has been obtained at another temperature. Epopt VALUE based expression is used to introduce the model, where the output voltage between terminals popt and 0 is $\operatorname{Popt}(\mathrm{T})$ value according (6), limited to values 0 and a maximum. The expression is then:

\section{Epopt popt 0 VALUE limit(Popt(T),0,max)}

To introduce the temperature dependency it is used the variable Tvar which will be used in the Pspice simulator to control the temperature values during its execution.

\section{3- Pspice model expression of Popt(T)}

According the theory exposed in section 2 it will be necessary to know at least the values of the threshold current $\mathrm{I}_{\text {th1 }}$ and $\mathrm{I}_{\text {th2 }}$ at two different temperatures $\mathrm{T}_{1}$ and $\mathrm{T}_{2}$ to calculate To according (7) and program the $\mathrm{I}_{\mathrm{th}}(\mathrm{T})$ variation expression (4), and values $\mathrm{SE}_{1}$ and $\mathrm{SE}_{2}$ to program the $\mathrm{SE}(\mathrm{T})$ expression (5), both to be introduced in (8). Based on it, Epopt expression is:

\section{Epopt popt 0 VALUE $\left\{\right.$ limit $\left.\left(\left(\left(S E_{1}+\left(T v a r-T_{1}\right) \cdot\left(\left(S E_{2}-S E_{1}\right) /\left(T_{2}-T_{1}\right)\right)\right) \cdot\left(I(V I d)-\left(I_{\text {th1 }} \cdot \exp \left(\left(T v a r-T_{1}\right) / T o\right)\right)\right)\right), 0, \max \right)\right\}$}

Temperatures used to measure the threshold current and the slope efficiency are not required to be the same. In the other hand, as laser diodes use to have limited operational temperature ranges, it is expected to obtain both values for $\mathrm{I}_{\text {th }}$ and $\mathrm{SE}$ at temperature values close to the upper and lower operational limits in order to minimize the ratio between a possible error in the measurements and the temperature variation.

\section{4- Characterization method}

Part of the electrical power used for feeding the laser diode device is converted in heat. In spite of using refrigeration systems, device's temperature increase during the measurement could modify the values of $\mathrm{I}_{\text {th }}$ and SE parameters intended to be measured. A way to avoid or minimize the temperature effect in the optical output power measurement is proposed, as a cold measurement method.

\section{1- Cold measurement method and characterization system setup}

The proposed characterization system obtains the relation between the Popt measured using a photodiode and the Ild current through the laser diode as a response to a fast rising up current slope. This performs a cold measurement method where the total current through the diode during the measurement period is very small and no temperature increase is provoked. Rising up slopes of only 20 $\mu$ s have been used with the proper current drivers, like the DAE-20050 of MONOCROM [13] with the mentioned single emitter laser diode of $8 \mathrm{~W}$ optical output power at $10.5 \mathrm{~A}$ of injected current according manufacturer test sheet, and with a C-mount $40 \mathrm{~W}$ optical output power laser bar diode at $40 \mathrm{~A}$. It means a total electrical charge through the diodes of $1.05 \cdot 10^{-4} \mathrm{C}$ and $4 \cdot 10^{-4} \mathrm{C}$ in each case at the end of the slope, when data of the last measure is acquired, and therefore no temperature increase is expected due to it. Because of this short time rising up slope, response curves need to be measured using fast data acquisition cards. In case of sampling a rising up slope of $20 \mu$ s the NI PCI-6115 [14] acquisition card is used. This card has $10 \mathrm{Ms} / \mathrm{s}$ per channel sampling speed, which allows the acquisition of about 200 samples per channel during that $20 \mu$ s slope.

The measurement and characterization system setup includes additionally temperature conditioning systems, and an integration sphere for Popt measurement stability avoiding laser beam directional effects. This integrating sphere is attenuating the optical power and therefore also allows the system to be used with high power laser diodes, which output cannot be directly connected to a photodiode. The integration sphere output power detected at the photodiode is calibrated in $\mathrm{CW}$ using an optical power meter measuring the laser diode output directly. Thermal optical power meters have been used with up to $40 \mathrm{~W}$ laser diodes, using laser thermal optical power sensors and power meters as described in the following Table 1.

Depending on the temperature conditioning system (climatic chamber, chiller, Peltier cells...) temperature will be measured using precision thermometers, thermistors or any other different system. It is known that the wavelength of the emitted light is changing with the temperature so, by supervising the spectrum width (FWHM parameter - Full Width at Half Maximum) and the wavelength value of the emitted light with spectrometers, any temperature drift during the measurement can be controlled. It is done in this setup schema by splitting the integration sphere output with optical fibres, to be measured at the photodiode and the spectrometer at the same time. Several spectrometers have been used as also shown in Table 1. 


\begin{tabular}{|c|c|c|c|c|c|c|}
\hline $\begin{array}{l}\text { Laser thermal } \\
\text { optical power } \\
\text { sensors }\end{array}$ & $\begin{array}{l}\text { Power } \\
\text { meters }\end{array}$ & Spectrometers & $\begin{array}{l}\text { Data } \\
\text { Acquisition } \\
\text { cards }\end{array}$ & $\begin{array}{c}\text { Maximum } \\
\text { Sampling speed }\end{array}$ & Divers & Photodiode \\
\hline $\begin{array}{c}\text { Ophir } \\
\text { 10A-PPS } 10 \mathrm{~W} \\
{[15]}\end{array}$ & $\begin{array}{l}\text { Ophir } \\
\text { Nova II } \\
{[17]}\end{array}$ & $\begin{array}{c}\text { Avantes } \\
\text { AvaSpec-2048- } \\
\text { SPU2 [18] }\end{array}$ & $\begin{array}{c}\text { NI USB-6009 } \\
{[21]}\end{array}$ & $\begin{array}{l}48 \mathrm{ks} / \mathrm{s} \text { (Divided } \\
\text { among channels) }\end{array}$ & $\begin{array}{l}\text { MONOCROM } \\
\text { DRVx2PITEC }\end{array}$ & $\begin{array}{l}\text { Thorlabs } \\
\text { PDA100A- } \\
\text { EC [22] }\end{array}$ \\
\hline \multirow[t]{2}{*}{$\begin{array}{c}\text { Ophir } \\
\text { 50A-PF-DIF-18 } 50 \\
\text { W [16] }\end{array}$} & $\begin{array}{l}\text { Ophir } \\
\text { Nova II }\end{array}$ & $\begin{array}{c}\text { StellarNet } \\
\text { Blue-Wave [19] }\end{array}$ & NI PCI-6115 & $\begin{array}{c}10 \mathrm{Ms} / \mathrm{s} \text { (Each } \\
\text { channel) }\end{array}$ & $\begin{array}{c}\text { MONOCROM } \\
\text { DAE-20050 }\end{array}$ & \\
\hline & & $\begin{array}{c}\text { HighFinesse } \\
\text { Angstrom LSA } \\
\text { (HF-LSA) [20] }\end{array}$ & & & & \\
\hline
\end{tabular}

\section{Table 1: Characterization system equipment}

Values measured at the photodiode, which corresponds to the optical output power, are compared with the related current through the laser diode measured at the control driver. Used Photodiode is the Thorlabs PDA100A-EC [22], which includes an adjustable voltage amplifier allowing compensation of the integration sphere attenuation. Popt and Ild values are compared using a data acquisition card in order to obtain its relation response curve. Two data acquisition cards have been used as shown in Table 1.

Fig.5 shows this setup schema and Fig.6 shows an image of the characterization system setup at the laboratory, using the DRVx2PITEC driver, the Blue-Wave spectrometer, the integration sphere, the NI USB-6009 data acquisition card and the Thorlabs

PDA100A-EC photodiode for the optical output power response characterization of the WSLX808008 laser diode.

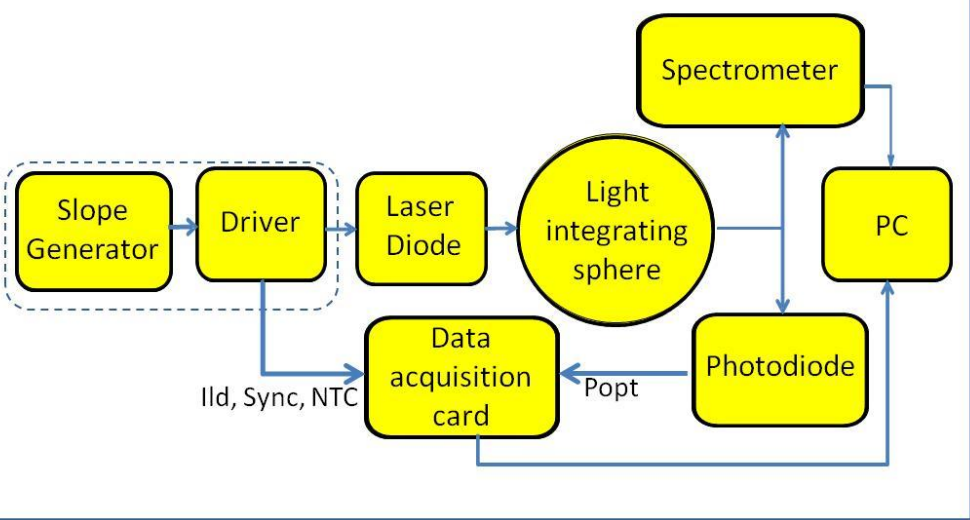

Fig.5: Characterization system schema 


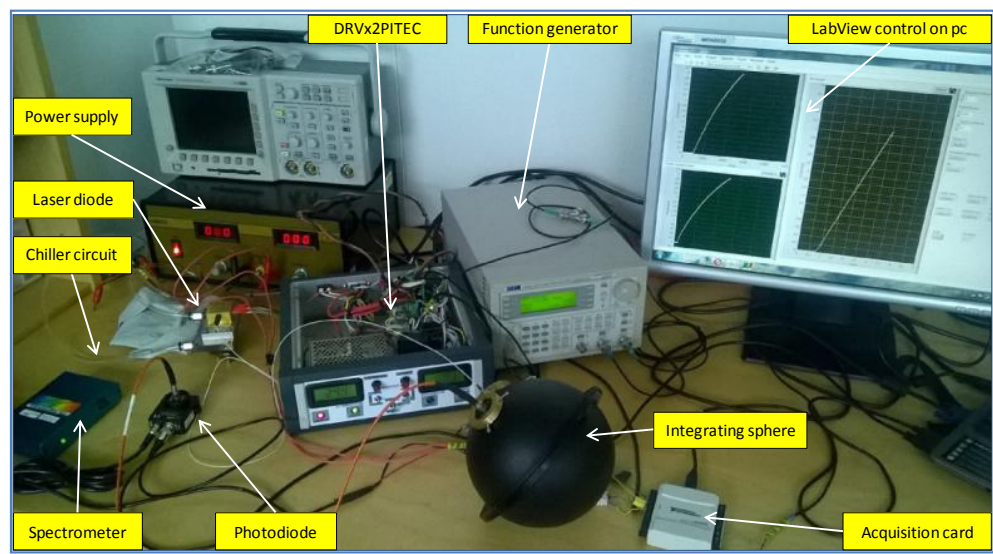

Fig.6: Characterization system setup image

\section{2- Automatic acquisition system}

In order to avoid any effect in the measurement due to delays between data acquisition card's channels, optimum case is using a card with independent channels and external trigger input. NI USB6009 and PCI-6115 data acquisition cards have been used. This second card has independent channels and a sampling speed up to $10 \mathrm{Ms} / \mathrm{s}$ per channel. An external trigger input is used to synchronize the sampling to acquire just the rising up slope, minimizing any possible heating effect in the measurement. A rising up slope of $20 \mu \mathrm{s}$ of Ild and Popt of the WSLX808008 laser diode sampled with this card, using the MONOCROM DAE-20050 driver. Resultant Popt/Ild chart are shown in Fig.7.

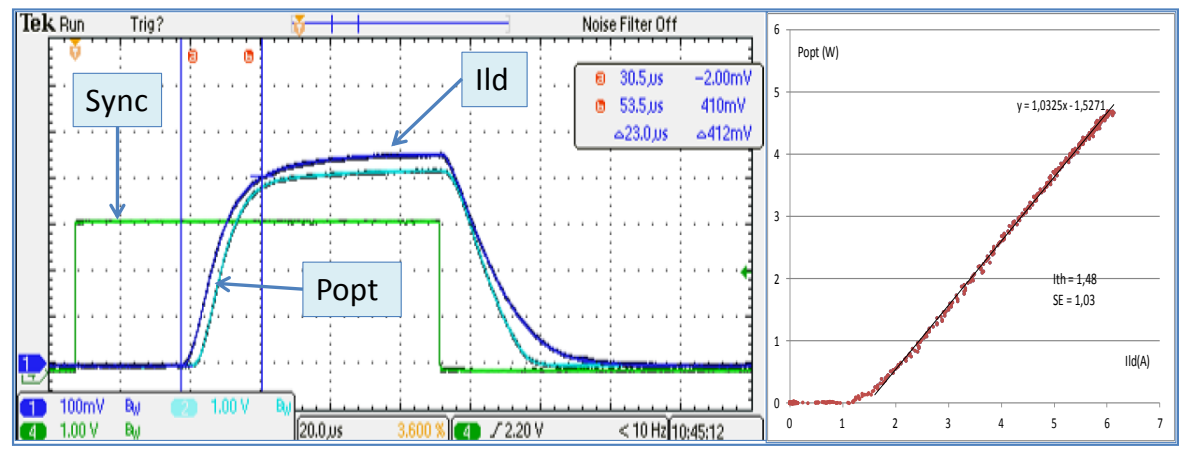

Fig.7: $20 \mu$ s rise up slope and Popt/Ild chart of the WSLX808008 laser diode

The data acquisition card is controlled with LabVIEW, used also to calculate the $\mathrm{I}_{\text {th }}$ and SE parameters from the obtained Popt/Ild relation at each temperature. LabVIEW control panel of the data acquisition system and calculated parameters can be seen at the following Fig.8. In this case, using the NI-USB6009 card and the MONOCROM DRVx2PITEC driver with same WSLX808008 laser diode device. This is the use case commented in the characterization system setup.

Optical output power detected at the photodiode is calibrated using a thermal optical power meter and introducing a correction factor. Current at the laser diode is read from a laser diode's serial shunt resistor in the driver. This signal is filtered and amplified to be correctly read at the data acquisition card adding a new correction factor. Correction factors are included in the LabVIEW calculation. Device temperature has been acquired from a laser device build-in NTC thermistor using a data acquisition card's third channel. $\mathrm{I}_{\mathrm{th}}$ and SE values are automatically calculated and stored periodically at intervals of $50 \mathrm{~s}$, together with the temperature at that moment. A rising up slope of $5 \mathrm{~ms}$ can be achieved with this driver. It corresponds to a periodic pulse of $5 \mathrm{~ms}$ and $0.01 \%$ duty cycle (T=50 s, Ton $=5 \mathrm{~ms}$ ) to avoid heating the diode. This allows to setup and automatic acquisition system to be used in climatic chambers using long period temperature conditioning cycles of several hours with slow temperature variations in order to allow temperature stability inside the device and to minimize any possible temperature gap between the semiconductor $\mathrm{p}$-n junction and the NTC thermistor where temperature is measured. System can be also used with other programmed temperature conditioning systems. 


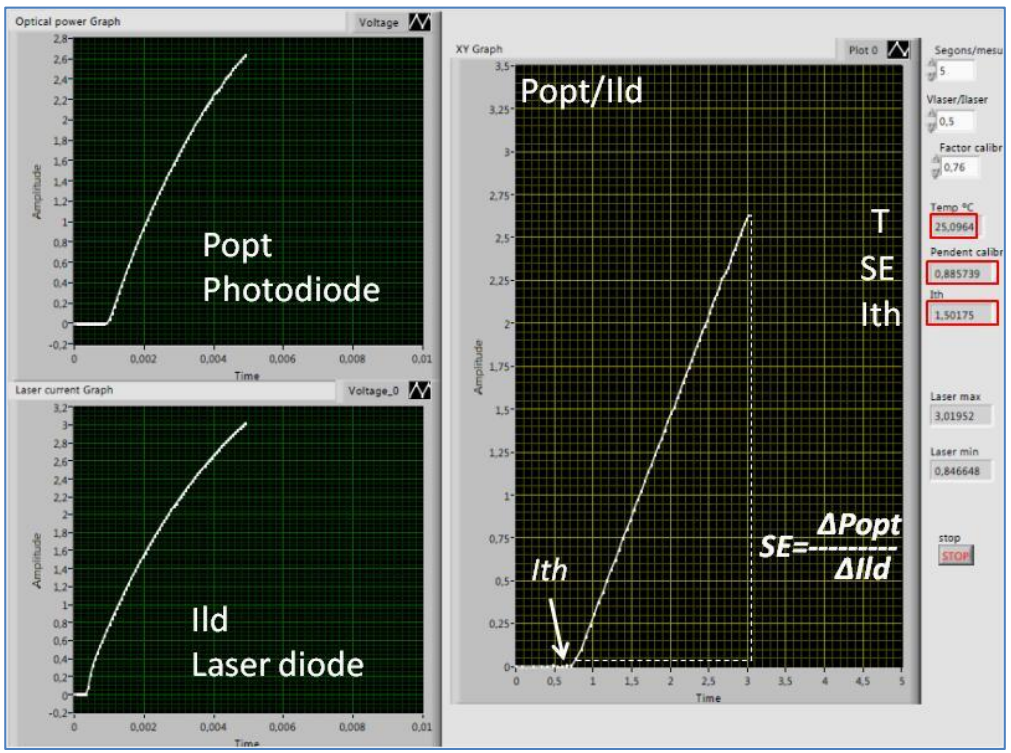

Fig.8: Data acquisition system. LabVIEW control panel

To confirm that no temperature drift is provoked during the measurement, emitted light spectrum width is controlled, as commented before. Following Fig.9 shows the emitted light spectrum during the measurement, in this case captured from the StellarNet BlueWave spectrometer control panel. Measured FWHM value is $2.99 \mathrm{~nm}$, which is not exceeding manufacturers specification value of 3 $\mathrm{nm}$, and therefore no wavelength drift is caused due to a temperature variation during the measurements.

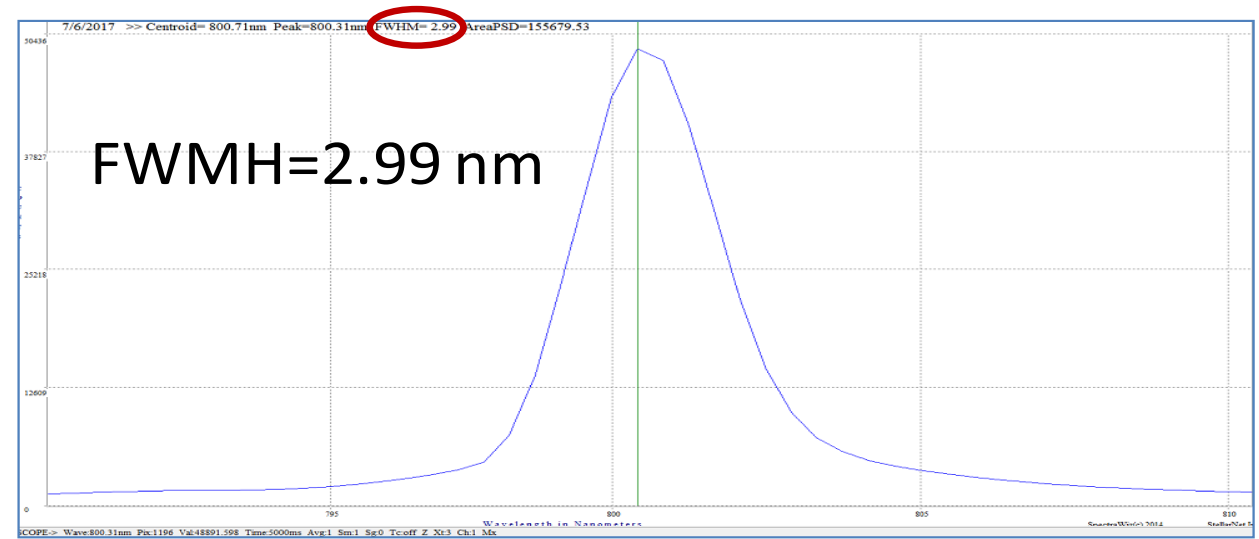

Fig.9 WSLX808008 laser diode spectrum during measurement at Blue-Wave spectrometer

\section{3- Model temperature parameters measurement}

$\mathrm{I}_{\mathrm{th}}(\mathrm{T})$ and $\mathrm{SE}(\mathrm{T})$ charts obtained in a climatic chamber of the WSLX808008 laser diode, with the described automatic acquisition system in a temperature range of $4{ }^{\circ} \mathrm{C}$ up to $31.5^{\circ} \mathrm{C}$, are shown in Fig.10. With measured $\mathrm{I}_{\mathrm{th}}(\mathrm{T})$ and $\mathrm{SE}(\mathrm{T})$ values at two different temperatures and according sections 1 and 2 the Pspice model can be written. 


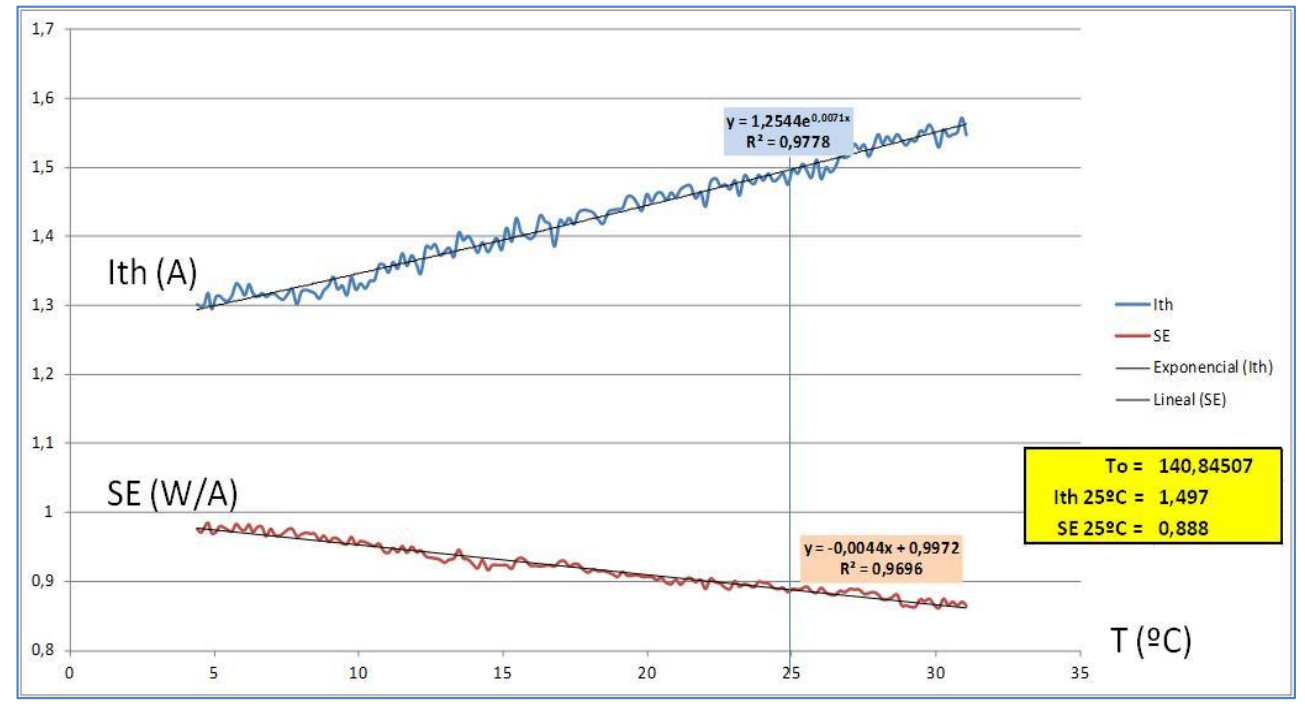

Fig.10: $I_{\text {th }}(T)$ and $S E(T)$ charts obtained at climatic chamber

To be mentioned that in this acquisition system setup the used photodiode is external to the laser diode device and mounted outside the climatic chamber, directly connected to the laser diode using an optical fibre. Only the laser diode part is inside the climatic chamber. Therefore there is no temperature variation at the photodiode and there is no temperature effect in its sensitivity. Photodiodes response is almost not dependent on temperature, but in this way it is assured that it should be no effect at all because of it. Even though the used device has an internal build-in photodiode, it has not been used because of the above explained reason.

As this is not a high power laser device, the laser diode output fibre has been able to be directly connected to the photodiode, and no integrating sphere has been used in this case to simplify the setup used at the climatic chamber. Optical output power detected at the photodiode has been calibrated using the thermal optical power measurements. Once confirmed with the spectrometer that no spectrum drift was existing with the test signal as commented previously, the spectrometer has not been neither used in the climatic chamber for the same reason. Fig. 11 shows an image of the automatic acquisition system setup at the climatic chamber.

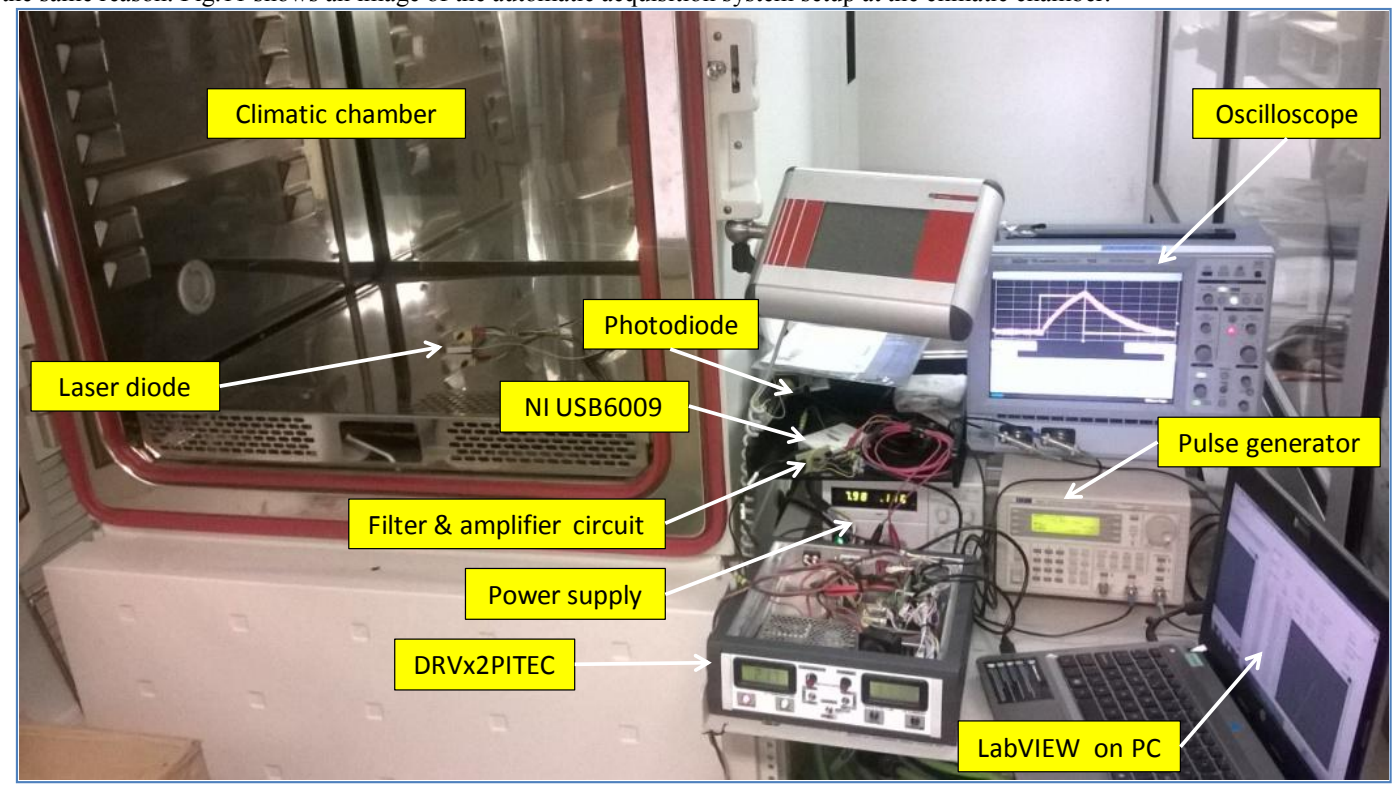

Fig.11: Automatic acquisition system setup at the climatic chamber

54.4- Theory and hypothesis confirmation. 
With the obtained results at the climatic chamber shown in Fig.10 it can be confirmed the theory laser diodes Popt(T) model is based on. In one hand and related to the $\mathrm{I}_{\text {th }}$ variation with the temperature, the obtained response curve shows an exponential increase behaviour according theory (4) where the exponent divider value To is $140.845 \mathrm{~K}$. This is the case of a AlGaAs laser diode of $808 \mathrm{~nm}$ wavelength and $8 \mathrm{~W}$ of optical output power. Similar values of To have been obtained in several laser diodes devices. For instance, $\mathrm{To}=130 \mathrm{~K}$ in a laser bar diode of same wavelength and $40 \mathrm{~W}$, and $\mathrm{To}=145 \mathrm{~K}$ in a AlGaInP laser diode device of $5 \mathrm{~mW}$ and $670 \mathrm{~nm}$ wavelength, the SLD1121VS from SONY [23]. Related to SE, the hypothesis is that the SE value is linearly decreasing with the temperature increase (5). This hypothesis is confirmed with the obtained data of this $8 \mathrm{~W}$ laser diode where a linear decrease of $\Delta \mathrm{SE}=$ $0.0044(\mathrm{~W} / \mathrm{A}) /{ }^{\circ} \mathrm{C}$ has been measured.

\section{5- Pspice programmed model}

Programmed model of the WSLX808008 laser diode based on the Pspice model schema exposed in section 3 is shown in the following Fig. 12. It is based on existing laser diodes components and in the diode available model in Pspice libraries. Regarding the diode model, with command "model laserdiode D" the Pspice diode library is used for the electrical response. Is, Rs and EG parameters values obtained in section 2 are introduced as specific values in the diode model. Other diode parameters are using library default values as those are not affecting the forward bias response where laser effect is produced. In case of parameter XTI, which is affecting the temperature dependent electrical response, default Pspice diode library value is XTI=3 for a p-n semiconductor diode, and it is also suitable for a laser diode because it is also a p-n semiconductor diode, so no need to specify a different value.

Only those components that are necessary for the optical power output specification have been kept in the model compared with available laser diodes programmed components in Pspice libraries. Photodetector diode has been deleted, as all current through its terminals in forward bias is specified by the current source, as commented before. Also other components like a dummy resistor have been deleted. The main contribution to the model is shown in the programmed model using bold fonts. It is related to the optical output power expression and its dependency on the temperature, the temperature variable introduction, and temperature parameters values. Deleted parts from the reference component model are also shown in bold fonts. The resultant Pspice programmed model is intended to only include the minimum necessary optical output power related parts.

Related to the optical output power parameters and their temperature dependency, used values of $\mathrm{I}_{\text {th }}$ and SE for the model have been obtained at $5{ }^{\circ} \mathrm{C}$ and $30^{\circ} \mathrm{C}$. At the climatic chamber it has been confirmed the correct operation of this laser diode device in the $4{ }^{\circ} \mathrm{C}$ up to $31.5^{\circ} \mathrm{C}$ temperature range, even though specification of this device [6] mentions a safer operation range of $10^{\circ} \mathrm{C}$ to $30^{\circ} \mathrm{C}$. Values of $\mathrm{I}_{\mathrm{th}}\left(5^{\circ} \mathrm{C}\right)=1.3 \mathrm{~A}, \mathrm{I}_{\mathrm{th}}\left(30^{\circ} \mathrm{C}\right)=1.55 \mathrm{~A}$ are measured and applying $(4) \mathrm{To}=140.845 \mathrm{~K}$, in this case obtained directly from the exponential expression of $\mathrm{I}_{\mathrm{th}}(\mathrm{T})$ chart measured at the climatic chamber and shown in Fig.10. Same temperature values are used for SE values, $\mathrm{SE}\left(5{ }^{\circ} \mathrm{C}\right)=0.975$ and $\mathrm{SE}\left(30{ }^{\circ} \mathrm{C}\right)=0.865$ which means a linear decrease of $\Delta \mathrm{SE}=-0.0044(\mathrm{~W} / \mathrm{A}) /{ }^{\circ} \mathrm{C}$. With those values the Popt $(\mathrm{T})$ expression to be introduced in the voltage controlled voltage source in the model (9) can be written. Photodiode in this case is limited to $15 \mathrm{~W}$ with a sensitivity of $1 \mathrm{~mA} / \mathrm{W}$. This value should be adapted to the used photodiode in a real circuit. Photodiode component is not included in the model, but only the Gpd source where this sensitivity is introduced as explained before. Tvar is used as the temperature variable, and with the Pspice command T_ABS=Tvar included in the laser diode model, temperature variation is only affecting the laser diode component. Tvar is initialised at $25{ }^{\circ} \mathrm{C}$ but temperature values will be specified during the simulation execution. 


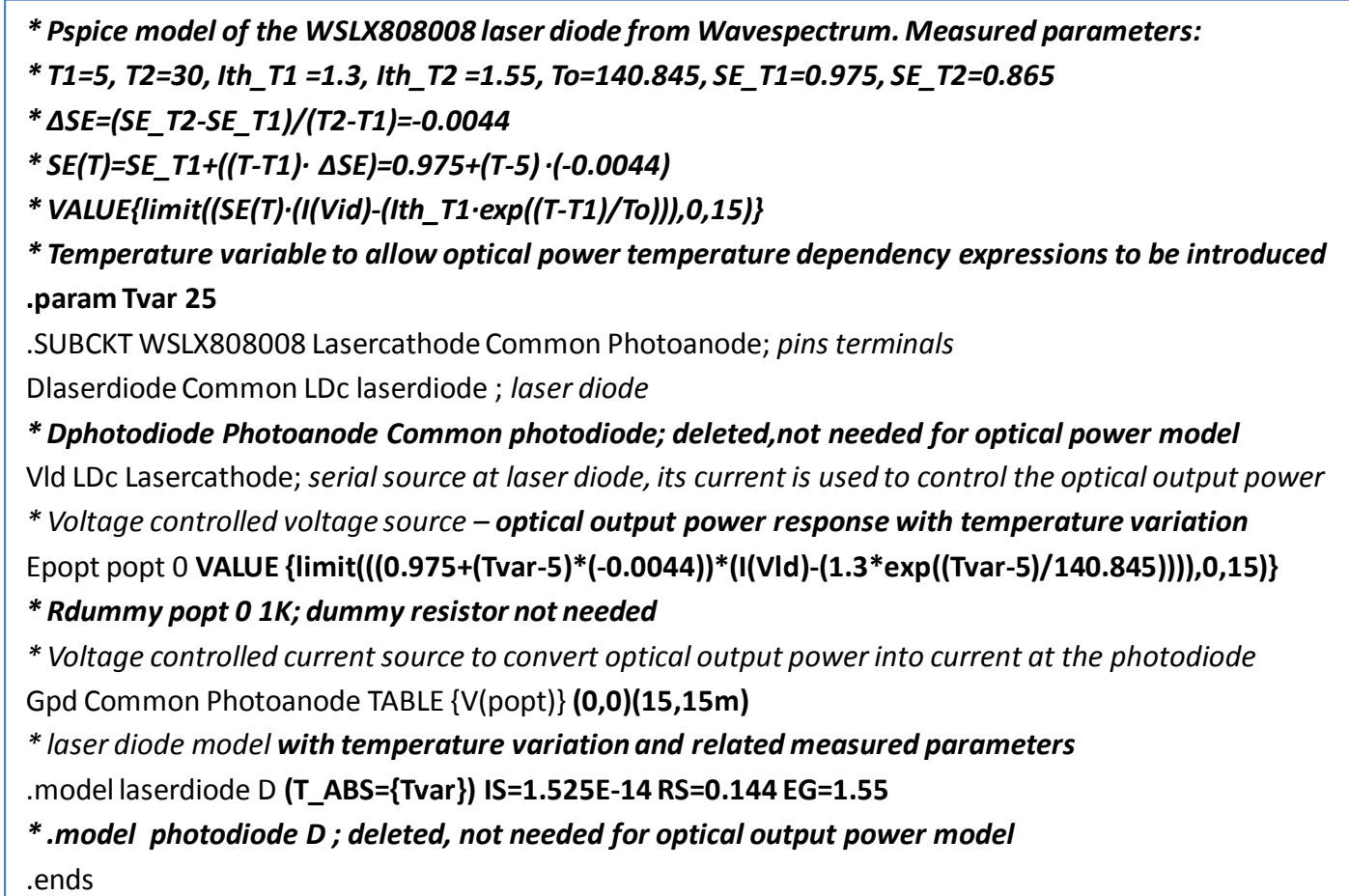

Fig.12: WSLX808008 laser diode Pspice model

\section{6- Simulation results. Model validation}

Simulation results of the programmed Pspice model proposed in Fig. 12 , in the $5{ }^{\circ} \mathrm{C}$ to $30{ }^{\circ} \mathrm{C}$ temperature range, at every $5{ }^{\circ} \mathrm{C}$ are shown in the following Fig. 13 . At $25^{\circ} \mathrm{C}$, simulation values results are $8 \mathrm{~W}$ of optical output power at $10.5 \mathrm{~A}$, and $\mathrm{I}_{\text {th }}=1.498 \mathrm{~A}$. Device test sheet values provided by manufacturer also at $25^{\circ} \mathrm{C}$ are $8 \mathrm{~W}$ at $10.5 \mathrm{~A}$ and $\mathrm{I}_{\text {th }}=1.5 \mathrm{~A}$. Measured values at $25^{\circ} \mathrm{C}$ shown in Fig. 8 are $\mathrm{I}_{\mathrm{th}}=1.5 \mathrm{~A}$ and optical output power at $10.5 \mathrm{~A}$ corresponding to the measured value of $\mathrm{SE}=0.886$ is also $8 \mathrm{~W}$. Therefore all values are matching and validating the model.
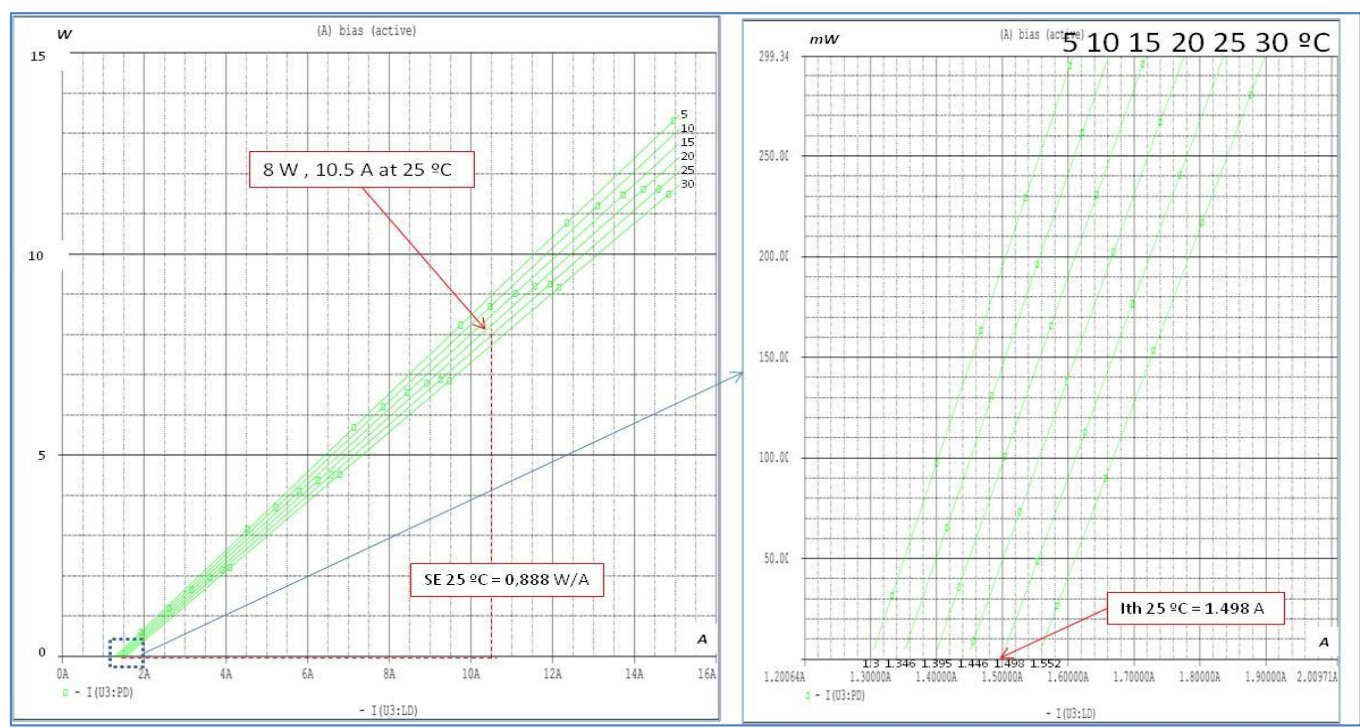

Fig.13: Pspice simulation results 


\section{5- Conclusions}

Proposed Pspice model schema and program allows an effective modelling of laser diodes optical output power. Characterization setup system including the cold measurement method and the automatic acquisition system with LabVIEW control allow quick and reliable measurements of the necessary parameters. Fast data acquisition cards with independent channels and external trigger inputs are recommended to be used in the characterization setup. Temperature drift control based on emitted light spectrum supervision using spectrometers is also recommended. Obtained charts of $\mathrm{I}_{\mathrm{th}}(\mathrm{T})$ and $\mathrm{SE}(\mathrm{T})$ are confirming the theory considered for the model, and especially, validating the hypothesis that $\mathrm{SE}(\mathrm{T})$ is linearly decreasing with the temperature increase. The modelling method has been used in laser diodes of $5 \mathrm{~mW}, 10 \mathrm{~mW}, 8 \mathrm{~W}$ and up to a $40 \mathrm{~W}$ laser bar diode. In this article the overall modelling method and characterization system is applied to a $8 \mathrm{~W}$ single emitter diode, the WSLX808008 from Wavespectrum, as example of application. Manufacturer provided data, measured values and simulated results are matching and therefore validating the modelling method and the obtained Pspice model.

\section{References}

[1] Zhu H, Ruset IC, Hersman FW (2005) Spectrally narrowed external-cavity high-power stack of laser diode arrays. Opt Lett 30:1342-1344

[2] Gerd Keiser, Semiconductors Laser Diodes. Lecture 13 pp. 37-38. http://studylib.net/doc/18066820/lecture-13-semiconductor-laser-diodes

[3] Paul D. Mitcheson. EE2.3: Semiconductors Modelling in Spice/PDm-v1.0. Spice Diode and BJT models. pp. 4-8. Department of Electrical and Electronic Engineering, Imperial College, London. http://www.imperial.ac.uk/people/paul.mitcheson/teaching

[4] J.S.Smith. Lecture 20: PN diodes (forward bias) small signal model, BJTs. pp.10-11(University of California, Berkeley) Department of EECS. Spring 2004.

[5] Pspice Reference Guide, Cadence Design Systems, Inc. Cadence PCB systems Division. May 2000

[6] WSLX-808-008-H-T Product Specification Wavespectrum Laser Inc. www.wavespectrum-laser.com

[7] Datasheet_DRVx2PITEC 15A Laser diode driver. Monocrom Electronics. www.monocrom.com

[8] User Guide_DRVx2PITEC. Monocrom Electronics, www.monocrom.com

[9] F. Schubert, Light-Emitting Diodes, 2nd ed. p. 207. (Cambridge University Press, Cambridge, UK, 2006)

[10] SANYO, DL-3149-057 specification, Ver.1, p-1-Jun. 2000. SANYO Electric Co., Ltd.

[11] SONY, SLD323V specification, E93207C19-PS, p-4. Sony Corporation

[12] HITACHI, HL6722G specification ADE-208-220C(Z) 4th Edition, p-4. Dec.2000, Hitachi Ltd.

[13] DAE-20050 Driver for High Power Laser Diodes. Monocrom electronics. www.monocrom.com

[14] NI PCI-6115 Multifunction I/O device. Datasheet \& Specification. www.ni.com

[15] 10A-PPS Laser Thermal Power Sensors. Low Power Thermal Sensors, www.ophiropt.com

[16] 50A-PF-DIF-18 Laser Thermal Power Sensors. Low Power Thermal Sensors, www.ophiropt.com

[17] Nova II Laser Power and Energy Meters, www.ophiropt.com

[18] AvaSpec-ULS2048 Datasheet. Avantes enlightening spectroscopy. www.avantes.com

[19] Blue-Wave Miniture Spectrometer Analytical Instrumentation SNI-PressRel.doc www.StellarNet-Inc.com

[20] HighFinesse LSA Laser Spectrum Analyser, www.highfinesse.com

[21] NI USB-6009 Multifunction I/O device. Datasheet \& Specification. www.ni.com

[22] Thorlabs PDA100A-EC Manual and product datasheet. Thorlabs Inc. US, www.thorlabs.com

[23] SONY, SLD1121VS specification, E93821C1X-PS, p-1. Sony Corporation.

[24] Dong Wook Lim, Hyung Uk Cho, Hyuk Kee Sung, and Jong Chang Yi, " A PSPICE Circuit Modeling of Strained AlGaInN Laser Diode Based on the Multilevel Rate Equations" Journal of the Optical Society of Korea Vol. 13, No. 3, September 2009, pp. 386-391. Hongik University, Seoul 121-791, Korea. Young Min Jhon Korea Institute of Science and Technology (KIST), Seongbuk, Seoul 136-791, Korea.

[25] Dan Alexandru Anghel, Andreea Rodica Sterian and Paul E. Sterian, "Modeling. Quantum Well Lasers". Hindawi Publishing Corporation Mathematical Problems in Engineering Volume 2012, Article ID 736529 , doi:10.1155/2012/736529. Academic Center for Optical Engineering and Photonics, Faculty of Applied Sciences, University "Politehnica" of Bucharest, 060042 Bucharest, Romania

[26] Jae Hong Kim, "Wide-Band and Scalable Equivalent Circuit Model for Multiple Quantum Well Laser Díodes". Georgia Institute of Technology. August 2005

[27] M.Lomer, Francisco J. Ruiz García. J.Echevarria, J.L.Arce, M.Morante. J.M. López-Higuera "Modelización y Simulacion de dispositivos optoelectrónicos utilizando el programa SPICE" URSI 97. II 453-456. ETSI Telecomunicación, Universidad de Cantabria. Departamento de Tecnología Electrónica - Grupo de Ingeniería Fotónica,

[28] Houssam Kanj, " Circuit-Level Modeling of Laser Diodes". Graduate Faculty of North Carolina State University. Electrical Engineering Raleigh May 2003.

[29] Houssam Kanj, " Double Heterojunction Laser Diode (Tucker Model) DHLD " NC State University, 2003

[30] M. S. Ozyazici, "The complete electrical equivalent circuit of a double heterojunction laser diode using scatterring parameters" Journal of Optoelectronics and Advanced Materials Vol. 6, No. 4, December 2004, p. 1243 - 1253. University of Gaziantep, Electrical and Electronics Eng. Dept., 27310 Gaziantep, Turkey

Formatted: English (United Kingdom)

Formatted: English (United Kingdom)

Formatted: English (United Kingdom)

Formatted: English (United Kingdom)

Formatted: English (United Kingdom)

Formatted: English (United Kingdom)

Formatted: English (United Kingdom)

Formatted: English (United Kingdom)

Formatted: English (United Kingdom) 
31] Vítězslav JEŘÁBEK ${ }^{1}$, Ivan HÜTTEL ${ }^{2}$, "Theoretical Model of the Bistable Semiconductor Laser Diode Based on the Rate Equations". RADIOENGINEERING, Vol. 20, NO. 2, June 2011.1-Department of Microelectronics, CTU FEE, Technicka 2, 166 27, Prague 6, Czech Republic. 2- Institute of Chemical Technology, Technicka 5, 166 28, Prague 6, Czech Republic

[32] Pablo Valente Mena, "CIRCUIT-LEVEL MODELING AND SIMULATION OF SEMICONDUCTOR LASERS". Department of Electrical and Computer Engineering University of Illinois at Urbana Champaign, 1998

[33] Lukasz Sliwczynski," Simple Spice model simulates laser diode". University of Mining and Metallurgy, Krakow, Poland EDN JULY 16, 1998

[34] Intusoft, " Modeling Lasers With IsSpice and PreSpice "Intusoft Newsletter. Personal Computer CircuitDesign Tools. July 1989

\section{Acknowledgment}

This work was funded by the Industrial Doctorates program from Generalitat de Catalunya and Agaur under contract "PROJECTE DE DOCTORAT INDUSTRIAL EXPEDIENT 2015 DI 004.". 